\title{
Medically Complex Children; Defining A Specialty
}

\author{
Mark Bleazard* \\ Pediatric Clinical Nurse Specialist, Nemours/Alfred I du Pont Hospital for Children, Delaware, USA \\ *Corresponding author: Mark Bleazard, Pediatric Clinical Nurse Specialist, Nemours/Alfred I du Pont Hospital for Children, \\ Delaware, USA. \\ To Cite This Article: Mark Bleazard. Medically Complex Children; Defining A Specialty. Am J Biomed Sci \& Res. 2019 - 4(2). AJBSR.MS.ID.000774. \\ DOI: 10.34297/AJBSR.2019.04.000774
}

Received: July 18, 2019 | Published: July 23, 2019

\section{Opinion}

Medically complex children can be defined as subset of pediatrics with multiple co-morbidities, technology dependence, increased healthcare needs, and significant functional limitations [1]. The proportion of medically complex children requiring services in the pediatric healthcare environment is rapidly increasing [1]. In addition, medically complex children have the potential to significantly impact institutional indicators of success including hospital-readmissions and mortality rates, as well as healthcare costs [2,3]. Significant vulnerability as well as increased reliance on artificial and technological devices for survival may also disproportionally impact other quality measures of success including nosocomial infections, ventilator associated pneumonia, central line associated blood stream infections, surgical site infections, catheter associated urinary tract infections, pressure ulcer rates, and sepsis morbidity and mortality rates.

As a result, comprehensive and expert care from clinicians that can provide additional surveillance to mitigate complications and to promote optimal patient outcomes is necessary. However, the term "medically complex" alone may be ambiguous and may not provide clinicians nothing more than perhaps several assumptions. Unlike other clinical specialties within pediatrics and healthcare, the care of medically complex children can be difficult to define as a specialty. Without adequate training and preparation, this uniquely vulnerable population is at even greater risk. Therefore, it is imperative that clinical leaders caring for medically complex children advocate for this population by first recognizing it as unique from general pediatrics of well-children, with whom have a separate set of risks and complications and define as a specialty alongside other well-established pediatric populations. This clear delineation establishes a foundation for further education and preparation of clinicians providing care to medically complex children.

Educators in the academic setting should include didactic content to establish the care of medically complex children as a specialty that compliment clinical experiences. Leaders within the clinical setting responsible for education, care management, and patient care outcomes must also facilitate evidence-based practice surrounding medically complex pediatric care provision. Content should include clinical management and review of the most frequently observed disorders associated with medically complex children, such as seizures, respiratory distress, feeding intolerance, technological dysfunction, peri-operative management, and musculoskeletal complications [1,2]. A review of common technological dependencies, including anti-epileptic devices such as vagal nerve stimulators, cerebrospinal fluid shunts, tracheostomy and ventilator support, central venous catheter reliance, enteral tube support, artificial genitourinary stomas, and musculoskeletal support devices including anti-spasmodic infusion implants is indicated [1,2]. Caregiver factors should also be reviewed and include burnout, family-centered care, resource management, alteration of family dynamics, palliative care, and end of life management [4]. System challenges, such as specialty ambiguity, clinician burnout or compassion fatigue, and reliance on the healthcare system or the medical environment for survival must be included in review.

Increasing complexity and prevalence of medically complex children within the healthcare environment, as well as the future direction of healthcare to provide value-based care associated with optimal patient care outcomes, necessitate that clinical leaders in academia as well as in the clinical setting provide adequate preparation to care for this unique and vulnerable population. A pre-requisite to the success of this endeavor, however, is clearly defining the disease prevention, management, and health promotion of medically complex children as a specialized provision of care requiring expert clinicians.

\section{References}

1. Russell C, Simon T (2014) Care of Children with Medical Complexity in the Hospital Setting. Pediatric Ann 43(7): e157-162.

2. Cohen E, Berry JG, Camacho X, Anderson G, Wodchis W, et al. (2012) Patterns and Costs of Health Care Use of Children with Medical Complexity. Pediatrics 130(6): e1463-1470.

3. House SA, Coon ER, Schroeder AR, Ralston SL (2017) Categorization of National Pediatric Quality Measures. Pediatrics 139(4): e20163269. 
4. Simon TD, Berry J, Feudtner C, Stone BL, Sheng X, et al. (2010) Children with Complex Chronic Conditions in Inpatient Hospital Settings in the
United States. Pediatrics 126(4): 647-655. 